\title{
Theranostics: A Way of Modern Medical Diagnostics and the Role of Chitosan
}

\author{
Tanvi Jain ${ }^{1,2}$, Sushil Kumar ${ }^{1}$ and Dutta PK $^{1,3^{*}}$ \\ ${ }^{1}$ Department of Chemical Engineering, Motilal Nehru National Institute of Technology, Allahabad-211004, Uttar Pradesh, India \\ ${ }^{2}$ Department of Chemistry, Motilal Nehru National Institute of Technology, Allahabad-211004, Uttar Pradesh, India \\ ${ }^{3}$ Centre for Medical Diagnostic \& Research, Motilal Nehru National Institute of Technology, Allahabad-211004, Uttar Pradesh, India \\ *Corresponding author: Dutta PK, Department of Chemistry, Centre for Medical Diagnostic \& Research, Motilal Nehru National Institute of Technology, \\ Allahabad-211004, Uttar Pradesh, India, Tel: +91 9936740953; Fax: 91-0532-2545341; E-mail: pkd_437@yahoo.com
}

Received date: February 13, 2015; Accepted date: February 23, 2015; Published date: March 02, 2015

Copyright: ( 2015 Jain T, et al. This is an open-access article distributed under the terms of the Creative Commons Attribution License, which permits unrestricted use, distribution, and reproduction in any medium, provided the original author and source are credited.

\begin{abstract}
The concept of therapeutic and diagnostic activities is mainly concerned to medical and pharmaceutical sciences. However, the recent trends earmarked the synergistic approaches of science and technology to provide more vibrant and effective medical treatment for the solution of health complications in medical sciences and it's the genesis of modern medical diagnostic. Hence, medical research is directed towards most promising and challenging fields of therapeutics + diagnostics $=$ theranostics. The feasibility of theranostics nowadays is apprehended in various disease diagnostics and curing processes. The modern medical diagnostic techniques are based on molecular imaging where each technique has its own characteristics, merits and demerits and most of the imaging modalities are complementary to each other. To provide better results of imaging the theranostic agents play very important role. The useful biopolymer research has been assisted towards the evolution of improved imaging agents in the form of quantum dots, nanoparticles and other new regenerative medication. The mini review focuses on theranostic development with special emphasis on quantum dots, and nanoparticles in the light of biopolymer, chitosan for cancer diseases.
\end{abstract}

Keywords: Therapeutics; Diagnostics; Quantum dots; Nanoparticles; Chitosan; Cancer

\section{Introduction}

The concept of personalized medicine has recently emerged as a promising way to address unmet medical needs especially in cancer treatments. The human system is so complicated that till one therapeutic agent is not enough to give the same effect in different patients with a same disease. Considering the inter-individual variability in therapeutic response, a patient-specific treatment may produce better therapeutic outcomes, while reducing patient discomfort and undesirable side-effects [1]. The word "theranostic" by coupling the techniques therapy and imaging was first used in 2002 by Funkhouser during the treatment of breast cancer [2]. Thus, theranostics deliver therapeutic drugs and diagnostic imaging agents at the same time within the same excipient. The concept of theranostics is a single platform with the combination of diagnostics and therapy emerged with progress in molecular imaging and nanomedicine in the form of new regenerative medicine. It also may be defined as a combination of nano based system in order to create new actualized methods of treatments with advanced efficacy for various diseases. In such approaches, medical treatments are altered to the precise features of individual patient [3]. By combining molecular imaging functionalities with therapy, a theranostic approach could be advantageous in identifying and selecting a particular subgroup of patients with a specific molecular phenotype.

In recent years, nano-theranostics involves the synthesis and application of numerous nanoparticle carriers, such as polymer systems, dendrimers, metal and non-metallic nanoparticles, polymeric nanocomposites and carbon nanotubes, for immediate, controlled and targeted delivery of therapeutic agents in order to achieve better results with fewer side effects [4]. Apart from controlling the release rate, these nano-regime particles can increase the surface area which allows for a high loading capacity of imaging probes, drugs, or targeting entities and also easily eject from blood vessels into malignant or damaged tissues due to cracked and erratic shapes of blood vessels at the tumour site and they can be retained at tumor sites having damaged lymphatic system of that site $[5,6]$. For theranostic purposes, enormous nanomaterials such as polymers and silica have been extensively investigated. The emerging applications of functionalized chitosan for imaging in the form of fluorescence imaging agents or as nanoscale carriers for delivery of therapeutic agents are amazing. The advantages of chitosan involve excellent biodegradability and biocompatibility also having low toxicity with numerous biological activities such as antimicrobial activity and low immunogenicity which provides bountiful opportunities for numerous applications in the field of tissue engineering, drug delivery, wound healing and other applications [7-13]. The present review will describe in brief the different imaging techniques, diagnostic agents and role of chitosan for the development of theranostics with reference to quantum dots, and nanoparticles for cancer treatment.

\section{Medical Imaging Techniques for Theranostics}

The main objective of the medical imaging is to analyse the bioprocesses non-invasively. It plays a significant role in handling the challenges of the characterization of biological processes at the cellular level in living objects.

Recently, numerous non-invasive imaging techniques may be used to envision the molecular targets in vivo like positron emission tomography (PET), ultrasound imaging (US), optical imaging, computed tomography (CT), single photon emission computed tomography (SPECT) and magnetic resonance imaging (MRI). 
These techniques consists of two types i.e., primarily molecular imaging techniques and primarily morphological/anatomical imaging techniques [14]. Primarily molecular imaging are characterized by high sensitivity, also include optical imaging and PET/SPECT; while, the second one are highlighted by high spatial resolution, like in CT, US and MRI $[14,15]$. Each procedure has its own properties in terms of its working, sensitivity, intricacy, period for data analysis and its cost.

Nowadays, integrated molecular/anatomical systems (e.g., SPECT/CT) have been adopted to combine the strengths of individual imaging techniques due to the fact that it can detect the molecular targets that are most important part in any of the disease identifying processes [16,17]. The different imaging modalities are generally considered complementary to each other $[18,19]$. The molecular imaging techniques with its characteristics, merits and demerits are shown in Figure 1.

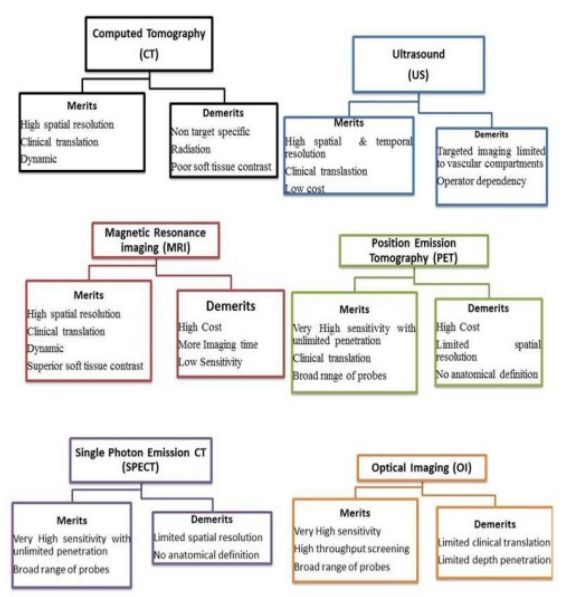

Figure 1: Various molecular imaging techniques with characteristics, merits and demerits.

\section{Basic Constituents for Fabrication of Theranostic Agents}

In medical diagnostic the contrast agents paly a very important role. Hence, designing of theranostic agents are very significant one. The main components of theranostic agents include payload carrier, payload, targeting ligands, signal emitters. The details constituents of any theranostic agents are explained in Figure 2.

The signal emitter consists of unique magnetic, optical, or radioactive properties. The drug may be any peptides, anti-cancer drugs, nucleic acids or therapeutic proteins where polymeric materials act as a drug carrier. Normally, modified polymers have its multifunctional groups like - $\mathrm{COOH},-\mathrm{SH},-\mathrm{OH}$ etc., which can provide multiple properties. Hence, both emitter and curative drugs can either be covalently conjugated to the surface of the drug carrier or noncovalently attached on theranostic carrier through aquaphobic or electrostatic interplay $[20,21]$.

Mostly, natural polymers having biodegradable properties are mostly used in biomedical practices like tissue engineering, wound healing, drug delivery etc., finally, targeting ligands with tremendous biocompatibility to tumor cells are always attached on the surface of the carriers [22]. Therefore, theranostics agents have enormous merits because they allow in situ targeted drug delivery, imaging and controlled release of drugs to tumor site.

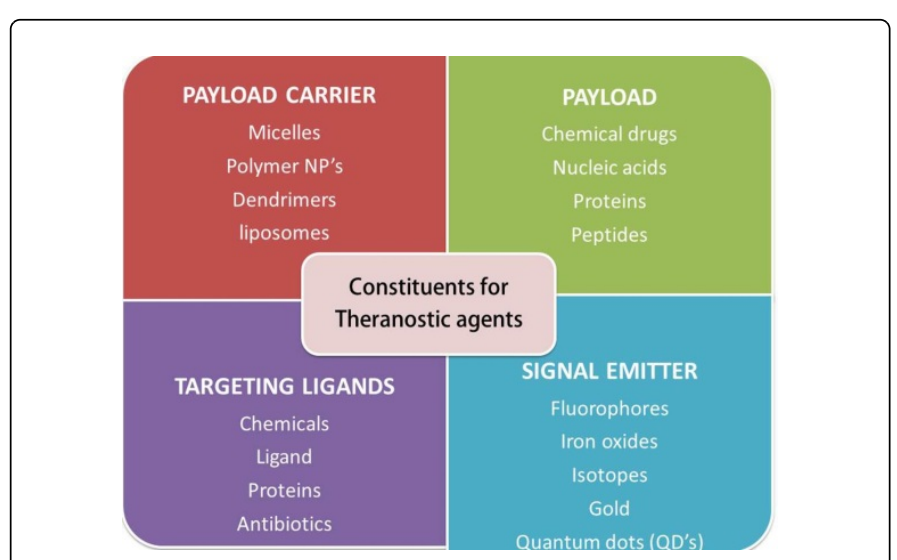

Figure 2: Basic constituents of theranostic agents.

\section{Medication with Theranostic Chitosan Based Quantum Dots (QDs)}

Fluorescent semiconductor nanocrystals, well known as quantum dots (QDs), are a breakthrough modality in the fields of theranostics. Their optical properties can be modified by varying its size of the particle and configuration. These inorganic fluorescent nanocrystals comprise groups of III-V (i.e. InP and InAs); II-VI (i.e. CdSe and $\mathrm{CdTe}$ ) semiconductor entities. The main drawback of QD's based theranostic carriers is its toxicity and irregularity $[23,24]$. The authors' laboratory has developed a highly luminescent chitosan-l-cysteine functionalized CdTe QDs film with the positive result of antibacterial study which reveals that the synthesized QDs based chitosan film is a promising candidate for wide range of biomedical applications [25].

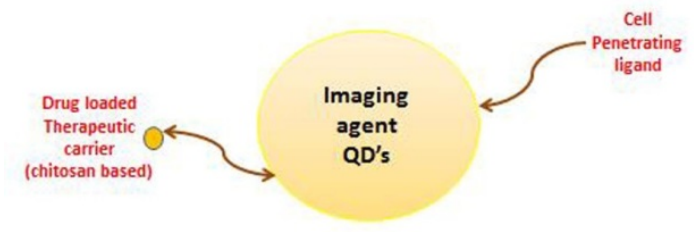

Figure 3: Illustration of a multimodal QD.

Ho et al. [26] displayed the intracellular detachment of polymer DNA complexes which further analysed by QD FRET (Fluorescence Resonance Energy Transfer). The researchers developed streptavidinfunctionalized, Cy5-labeled chitosan which was combined to QD- 
labeled pDNA through chemical bonding between biotin streptavidin. QD FRET studies was also studied using confocal microscopy at different time intervals after transfection in Human Embryonic Kidney 293 (HEK293) cells to study unpackaging of chitosan -p DNA aggregates. These analysis indicates that at $24 \mathrm{~h}$ after transfection, polyplexes were bounded in the perinuclear domain, whereas at $72 \mathrm{~h}$ post-transfection, polyplexes were disengaged and DNA was located in the nucleus without any signal emitted from chitosan (Cy5) [26].

Figure 3, shows the illustration of QD which serves as both a diagnostic agent (imaging) and a nanocomposites to incorporate multiple functional techniques, like a targeting ligand (peptide, antibody, or protein) and a therapeutic carrier.

In another study, Qiang et al. [27] prepared CdSe/ZnS quantum dot-encapsulated chitosan hybrid nanospheres (CS-QD) and then internalized by diseased tumor cells and it behaves like a labelling agent in cell imaging performed by confocal microscopy. CS-QD hybrid nanospheres also analysed for tumor in mice and accrued at tumor site by the route of blood circulation based on intravenous injection. Chitosan nanospheres will give the protection in both colloidal and optical stability arising from QDs. Likewise, the encapsulated QDs will give fluorescence on polymer nanospheres and thus polymer nanospheres will appear in cells and bodies.

Ma et al. [28] fabricated carboxymethyl chitosan-CdTe quantum dots. carboxymethyl chitosan on the surface of CdTe QDs had strong binding ability with $\mathrm{Zn}^{2+}$ results in the advancement of the fluorescence of CdTe QDs. Wee et al. [29] synthesised chitosan encapsulated QDs to deliver human epidermal growth factor receptor 2 (HER2) si-RNA to MCF-7 (human breast adenocarcinoma-7) and SKBR3 - human breast cancer cell line cells, where, the surface of the QD-chitosan composite was altered with the help of HER2 antibodies to attain targeted delivery of si-RNA to HER2 receptors. SKBR3 cells presented a larger number of HER2 receptors and as a consequence, more internalization was found in SKBR3 cells when distinguished with MCF-7 cells. After comparison with the control, HER2 gene expression levels were reduced to $80 \%$ when QD-chitosan composite was used to deliver HER2 si-RNA.

In another approach, Yuan et al. [30] synthesised blue-light emitting $\mathrm{ZnO}$ quantum dots with chitosan for targeted drug delivery specifically for tumours and they proposed that new generation of QDs with anti-cancer drug encapsulated with biocompatible polymer like chitosan provides a great platform to deliver tumor-targeted drugs and its analysis. Nontoxic water-soluble $\mathrm{ZnO}$ QDs with long haul fluorescence activity were also prepared by a chemical method using hydrolysis, encapsulated with chitosan and loaded with anti-cancer drug in which chitosan upgraded the stability of prepared quantum dots and thus can provide a better drug release nanocarrier for targeted drug delivery diagnosis as well as its treatment [30]. Thus, QDs had proved themselves as an impressive imaging agent for in vitro and in vivo purposes.

\section{Medication with Theranostic Chitosan Based NP's}

There has been colossal number of researches to explain polymerbased (like PEG, PLGA, Hyaluronic acid (HA) etc.) theranostic agents by characteristics of advantageous encapsulation properties of polymers. Presently, variety of chitosan based nanoparticles is widely in use for the cancer therapy, drug delivery and its diagnostics purposes. Yoon et al. [31] prepared glycol chitosan (GC)-based nanoparticles (CNPs) serve as useful nanocarriers that can effectively entrap both chemotherapy drugs and si-RNA to attain maximum efficacy by overcoming resistance. Doxorubicin, DOX-encapsulated CNPs (DOX-CNPs) or Bcl-2 si-RNA-encapsulated CNPs (si-RNACNPs) was evaluated and their physiochemical properties, including size, surface properties and $\mathrm{pH}$ sensitive behavior were studied, regardless of the different physical features of DOX and Bcl-2 si-RNA and the researcher confirms that CNP platform applied to two different types of drugs results in similar in vivo bio distribution and chemical kinetics, embellishing treatment in a dose-dependent manner [31]. In another study, $\mathrm{Na}$ et al. [32] fabricated chitosan nanoparticles which carry cyanine dye, Cy 5.5 for live imaging and nanoparticles loaded with paclitaxel for cancer treatment. Their work investigated glycol chitosan NPs with hydrophobic $5 \beta$-cholanic acid after chemical modification to confer the confined area of nanocarrier for encapsulation of the drug. As a result it was proposed that the remarkable tumor-targeting capability in numerous tumor models discovered from main factors as in vitrolin vivo stability, its contort, hasty uptake by target tumor cells. The outcome of this study suggested that the tumor-targeting capability was facilitated by long circulation and invasion through angiogenic vessels in tumor tissue. In 2013, Srinivasan et al. [33] described the synthesis of infrared dye, IR820-chitosan conjugates, potentials multipurpose imaging system for hyperthermia use in cancer treatments. These nanoconjugates had been formed by covalent reaction of chitosan to a carboxyl derivative IR820, and its optical imaging and hyperthermia uses. IR820-chitosan conjugates were able to achieve heat upon exposure through laser and produce hyperthermic cell growth inhibition in cancer cell lines in MES-SA (human sarcoma cell line), SKOV-3 and Dx5 (antibody reagent). The comparative evaluation also reported that amount of reticence of the cell growth due to hyperthermia was notably more in IR820 chitosan than IR820 which is present in MES-SA/Dx5 cells. Recently, Zhao et al. synthesised mesostructured $\mathrm{Fe}_{3} \mathrm{O}_{4} /$ chitosan nanoparticles along with doxorubicin (DOX) as a drug was delivered. Experimental results confirm that the synthesized mesostructured $\mathrm{Fe}_{3} \mathrm{O}_{4} /$ chitosan is a nimble nanocarrier for delivery of drugs owing to both its $\mathrm{pH}$-related properties and efficiency [34]. In another research, Lianjiang et al. [35] synthesised nanoscaled light-triggered nitric oxide (NO) carrier using chitosan and S-nitrosothiols and then encapsulated silver sulfide quantum dots ( $\mathrm{Ag}_{2} \mathrm{~S}$ QDs). The cell imaging was tested successfully, indicated that the $\mathrm{Ag}_{2} \mathrm{~S}$-CS-SNO nanospheres could emit good amount of NIR fluorescence and release NO in living cells for disease treatments [35].

Using different approach, lanthanide loaded iron oxide doped with chitosan nanoparticles were synthesised its optical imaging ability was imitated from doping of photostable lanthanide ions e.g. $\mathrm{Eu}^{3+}$ and $\mathrm{Tb}^{3+}$. In the new method, lathanide ions were taken rather than any QDs which might cause photo bleaching effect due to interaction with solvent entities and reactive molecules such as oxygen or ions dissolved in solution when those are exposed to a variety of tough environment [36]. Maria et al. [37] developed chitosan colloidal nanocarriers for the controlled delivery of drug docetaxel and reported that docetaxel remains completely effective during its encapsulation into the nanocarriers and thus nanocarriers transported docetaxel into cancer cells actively which resulted in a momentous increment in its antiproliferative properties which can really be useful in the field of theranostics [37]. 


\section{Conclusion}

In this review, recent effort in the evolving field of theranostics, we have mentioned chitosan theranostic agents in different formulations like nanoparticles and quantum dots. Due to the biological properties of chitosan, a lot of work can be done in the field of medical diagnosis for various disease treatments. Researchers have created chitosan based nanoparticle and quantum dots systems that can be used for cancer therapy, for transfer of anticancerous drugs and indicative imaging agents. The emerging applications of functionalized chitosan/ chitin for imaging in the form of fluorescence contrast agents or as nanoscale vehicles for delivery of therapeutics, is a great area that can be used for various biomedical applications. The evolution of nanotechnology has proposed a great breakthrough to promote the development of innovative theranostics. After inclusive analysis on immunotoxicity and cytotoxicity of theranostics agents the inclusion of theranostics into routine health care and diagnostic may thus become possible as a substantial tool of embodied and therapeutic medication.

\section{Acknowledgements}

One of the authors (TJ) wishes gratefulness to Dr. Sadhana Sachan, Head of the Chemical Engineering Department and Director Prof. P. Chakrabarti, Motilal Nehru National Institute of Technology, Allahabad, India, for providing the stipend, TEQIP II Grant of the Institute in the form of financial support for the research work.

\section{References}

1. Kalia M (2013) Personalized oncology: recent advances and future challenges. Metabolism 62 Suppl 1: S11-14.

2. Funkhouser J (2002) Reintroducing pharma: Theranostic revolution Curr. Drug Discovery 2.

3. Jokerst JV, Gambhir SS (2011) Molecular imaging with theranostic nanoparticles. Acc Chem Res 44: 1050-1060.

4. Muthu MS, Leong DT, Mei L, Feng SS2 (2014) Nanotheranostics application and further development of nanomedicine strategies for advanced theranostics. Theranostics 4: 660-677.

5. Maeda H, Nakamura H, Fang J (2013) The EPR effect for macromolecular drug delivery to solid tumors: Improvement of tumor uptake, lowering of systemic toxicity, and distinct tumor imaging in vivo. Adv Drug Deliv Rev 65: 71-79.

6. Maeda H (2012) Macromolecular therapeutics in cancer treatment: the EPR effect and beyond. J Control Release 164: 138-144.

7. Freier T, Koh HS, Kazazian K, Shoichet MS (2005) Controlling cell adhesion and degradation of chitosan films by $\mathrm{N}$-acetylation. Biomaterials 26: 5872-5878.

8. Dutta J, Chattopadhyaya MC, Tripathi VS, Dutta PK (2004) Chitin \& Chitosan: Novel biomaterials waiting for future developments. J Polym Mater 21: 321-332.

9. Rinaudo M (2008) Main properties and current applications of some polysaccharides as biomaterials. Polym Int 57: 397-430.

10. Jain T, Dutta PK (2011) Chitin Nanoparticles for Drug Delivery: Preparation, characterization and evaluation Asian Chitin J 7: 13-18.

11. Dutta PK, Dutta J, Tripathi VS (2004) Chitin and chitosan: Chemistry, properties and applications. Journal of Scientific and Industrial Research 63: 20-31

12. Dutta J, Dutta PK (2005) Chitin and chitosan: Opportunities \& challenges. SSM International Publication, Midnapore, WB

13. Yi H, Wu LQ, Bentley WE, Ghodssi R, Rubloff GW, et al. (2005) Biofabrication with chitosan. Biomacromolecules 6: 2881-2894.
14. Kircher MF, Hricak H, Larson SM (2012) Molecular imaging for personalized cancer care. Mol Oncol 6: 182-195.

15. Huang Y, He S, Cao W, Cai K, Liang XJ (2012) Biomedical nanomaterials for imaging-guided cancer therapy. Nanoscale 4: 6135-6149.

16. de Smet M, Langereis S, van den Bosch S, Bitter K, Hijnen NM, et al. (2013) SPECT/CT imaging of temperature-sensitive liposomes for MRimage guided drug delivery with high intensity focused ultrasound. J Control Release 169: 82-90.

17. John AE, Luckett JC, Tatler AL, Awais RO, Desai A, et al. (2013) Preclinical SPECT/CT imaging of av $\beta 6$ integrins for molecular stratification of idiopathic pulmonary fibrosis. J Nucl Med 54: 2146-2152.

18. Willmann JK, van Bruggen N, Dinkelborg LM, Gambhir SS (2008) Molecular imaging in drug development. Nat Rev Drug Discov 7: 591-607.

19. Shi Z, Neoh KG, Kang ET, Shuter B, Wang SC, et al. (2009) (Carboxymethyl)chitosan-modified superparamagnetic iron oxide nanoparticles for magnetic resonance imaging of stem cells. ACS Appl Mater Interfaces 1: 328-335.

20. Veiseh O, Gunn JW, Zhang M (2010) Design and fabrication of magnetic nanoparticles for targeted drug delivery and imaging. Adv Drug Deliv Rev 62: 284-304.

21. Lewandrowski KU, Gresser JD, Wise DL, Trantol DJ (2000) Bioresorbable bone graft substitutes of different osteoconductivities: a histologic evaluation of osteointegration of poly(propylene glycol-cofumaric acid)-based cement implants in rats. Biomaterials 21: 757-764.

22. Bamrungsap S, Zhao Z, Chen T, Wang L, Li C, et al. (2012) Nanotechnology in therapeutics: a focus on nanoparticles as a drug delivery system. Nanomedicine (Lond) 7: 1253-1271.

23. Zhu ZJ, Yeh YC, Tang R, Yan B, Tamayo J, et al. (2011) Stability of quantum dots in live cells. Nat Chem 3: 963-968.

24. Medintz IL, Uyeda HT, Goldman ER, Mattoussi H (2005) Quantum dot bioconjugates for imaging, labelling and sensing. Nat Mater 4: 435-446.

25. Kumar H, Srivastava R, Dutta PK (2013) Highly luminescent chitosan-Lcysteine functionalized CdTe quantum dots film: synthesis and characterization. Carbohydr Polym 97: 327-334.

26. Ho YP, Chen HH, Leong KW, Wang TH (2006) Evaluating the intracellular stability and unpacking of DNA nanocomplexes by quantum dots-FRET. J Control Release 116: 83-89.

27. Ma Q, Lin ZH, Yang N, Li Y, Su XG (2014) A novel carboxymethyl chitosan-quantum dot-based intracellular probe for $\mathrm{Zn} 2+$ ion sensing in prostate cancer cells. Acta Biomater 10: 868-874.

28. Lin Y, Zhang L, Yao W, Qian H, Ding D, et al. (2011) Water-soluble chitosan-quantum dot hybrid nanospheres toward bioimaging and biolabeling. ACS Appl Mater Interfaces 3: 995-1002.

29. Tan WB, Jiang S, Zhang Y (2007) Quantum-dot based nanoparticles for targeted silencing of HER2/neu gene via RNA interference. Biomaterials 28: 1565-1571.

30. Yuan Q, Hein S, Misra RD (2010) New generation of chitosanencapsulated $\mathrm{ZnO}$ quantum dots loaded with drug: synthesis, characterization and in vitro drug delivery response. Acta Biomater 6: 2732-2739.

31. Yoon HY, Son S, Lee SJ, You DG, Yhee JY, et al. (2014) Glycol chitosan nanoparticles as specialized cancer therapeutic vehicles: sequential delivery of doxorubicin and Bcl-2 siRNA. Sci Rep 4: 6878.

32. Na JH, Koo H, Lee S, Min KH, Park K, et al. (2011) Real-time and noninvasive optical imaging of tumor-targeting glycol chitosan nanoparticles in various tumor models. Biomaterials 32: 5252-5261.

33. Srinivasan S, Manchanda R, Fernandez-Fernandez A, Lei T, McGoron AJ (2013) Near-infrared fluorescing IR820-chitosan conjugate for multifunctional cancer theranostic applications. J Photochem Photobiol B 119: 52-59.

34. Guanghui Z, Jianzhi W, Xiaomen P, Yanfeng L, Xuemei Y, et al. (2014) Facile Solvo thermal Synthesis of Mesostructured Fe3O4/Chitosan Nanoparticles as Delivery Vehicles for $\mathrm{pH}$-Responsive Drug Delivery and Magnetic Resonance Imaging Contrast Agents. Chem Asian J 9: 546-553. 
Citation: Jain T, Kumar S, Dutta PK (2015) Theranostics: A Way of Modern Medical Diagnostics and the Role of Chitosan. J Mol Genet Med 9: 159. doi:10.4172/1747-0862.1000159

Page 5 of 5

35. Lianjiang T, Ajun W, Huili L (2013) Ag2S Quantum Dots Conjugated Chitosan nanospheres toward Light-Triggered Nitric Oxide Release and Near-Infrared Fluorescence Imaging. Langmuir 29: 15032-15042.

36. Wang W, Jiang X, Chen K (2012) Lanthanide-doped chitosan nanospheres as cell nuclei illuminator and fluorescent nonviral vector for plasmid DNA delivery. Dalton Trans 41: 490-497.
37. Maria VL, Daniel T, Dolores T, Anxo V, Fernando D, et al. (2009) Highly Efficient System To Deliver Taxanes into Tumor Cells: Docetaxel-Loaded Chitosan Oligomer Colloidal Carriers. Biomacromolecules 9: 2186-2193. 